\title{
More on the pulsation period of the cool component in the symbiotic binary AG Dra
}

\author{
M. Friedjung ${ }^{1}$, R. Gális ${ }^{2,3}$, L. Hric ${ }^{4}$, and K. Petrík ${ }^{5,6}$ \\ ${ }^{1}$ Institut d'Astrophysique, 98 bis Boulevard Arago, 75014 Paris, France \\ ${ }^{2}$ Faculty of Sciences, University of P. J. Šafárik, Moyzesova 16, 04154 Košice, The Slovak Republic \\ e-mail: galis@kosice.upjs.sk \\ 3 Partizánske Observatory, PO Box 59, 95801 Partizánske, The Slovak Republic \\ e-mail: hvezdap@nextra.sk \\ 4 Astronomical Institute of the Slovak Academy of Sciences, 05960 Tatranská Lomnica, The Slovak Republic \\ e-mail: hric@ta3.sk \\ 5 Department of Physics, Faculty of Education, Trnava University, 91843 Trnava, The Slovak Republic \\ e-mail: astropet@ta3.sk \\ ${ }^{6}$ Observatory and Planetarium, Sládkovičova 41, 92001 Hlohovec, The Slovak Republic
}

Received 12 March 2002 / Accepted 8 November 2002

\begin{abstract}
We have combined recent radial velocity measurements of Fekel et al. (2000) with previously studied older ones, to test for the continued presence of the second period, found by Gális et al. (1999), which could be due to pulsations of the cool component of the AG Dra binary system. Fekel's data, even taken by themselves, also marginally suggest the presence of variations near the previously found second period. The period is in any case clearly seen, when all radial velocity measurements are combined.
\end{abstract}

Key words. stars: binaries: symbiotic - stars: individual: AG Dra

\section{Introduction}

AG Dra is a symbiotic binary, containing a fairly cool giant of spectral type K4 or earlier (Kenyon \& Fernandez-Castro 1987 ) with a luminosity class rather of Ib or II according to Zhu et al. (1999). The other component is believed to be a hot white dwarf. Parameters for this system were derived by Mikolajewska et al. (1995). This object passes through quiescent and active stages; the latter may be explained by increased thermonuclear burning on a white dwarf, accreting matter from the wind of the cool component.

In previous studies a second period in addition to the orbital period of 549.7 days was found for the symbiotic binary AG Dra. This period of about 350 days, indicated in our work using photometry (Friedjung et al. 1998; Petrík et al. 1998), is somewhat less than the photometric second period of 378.5 days found by Bastian (1998). Our later study of radial velocities (Gális et al. 1999) indicated also a second period of 355.3 days. It was suggested that the second period could arise from pulsations of the cool giant component of the binary. Moreover it turned out that the epochs of major symbiotic activity started at times when the variations of radial velocity with

Send offprint requests to: M. Friedjung,

e-mail: fried@iap.fr both periods had simultaneous negative maxima. An explanation was suggested by Gális et al. (1999), involving non-radial pulsations of the cool component, leading to a higher accretion rate of the white dwarf component at certain times.

Recently Fekel et al. (2000) used Fourier transform spectra to measure high precision radial velocities of AG Dra. They did not confirm in their data the presence of the second pulsation period discovered in our previous paper (Gális et al. 1999). In this paper we shall re-analyse the various radial velocity data sets, including those of Fekel et al. (2000), in order to rediscuss the controversial conclusion published by these authors.

\section{Observations}

The photoelectric observational material used for the argument of this paper is the same as that already used and discussed in our previous paper (Gális et al. 1999). The results of recent photometric monitoring of this system will be the subject of our next paper.

The spectroscopic observational material consisting of 83 radial velocity values with a relatively high precision, discussed in this paper, has been extracted from Mikolajewska et al. (1995), Smith et al. (1996), Tomov \& Tomova (1997) and Fekel et al. (2000). All radial velocities have been determined using spectral absorption lines formed in the atmosphere of the 


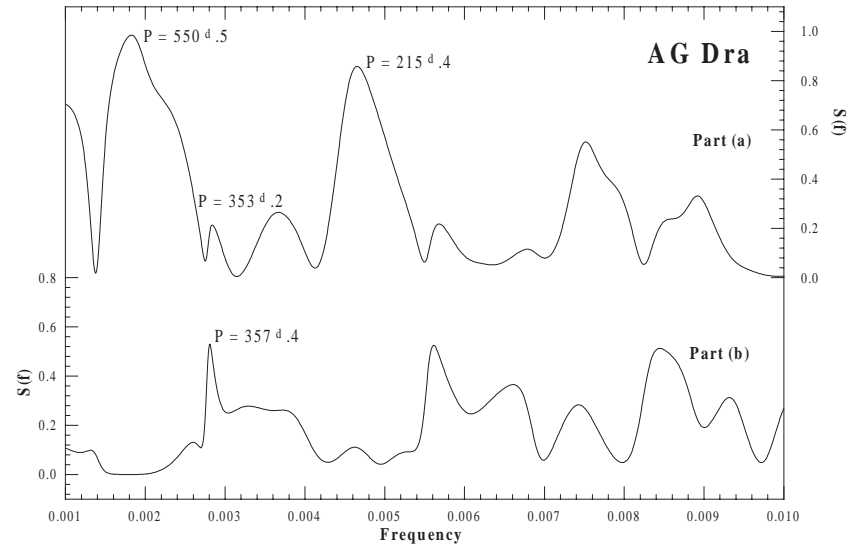

Fig. 1. Periodograms of AG Dra taken from data by Fekel et al. (2000): original data - Part a) and orbital response removed from the data Part b).

red giant. The data cover incompletely the time interval from JD 2446578.500 to JD 2451676.869 , but with good oversampling of individual sets.

\section{Analysis}

If we examine the observations of Fekel et al. (2000), we see that they were obtained over almost two orbital cycles or, in other words, only during 3 years at similar phases of the second period given by Gális et al. (1999). The phases of observations are between 0.36 and 0.59 (see in Fig. 5), with predicted second period radial velocity contributions between -1.0 and $-1.6 \mathrm{~km} \mathrm{~s}^{-1}$. This range is the same as the standard error of a single observation given by Fekel et al. (2000). Clearly it is neither easy to detect pulsations in such conditions nor to prove their reality. The reason is that the second period is close to one year and the data have been obtained in the same observing season. On the other hand the observations used by Gális et al. (1999) were secured over a time span of 13 years, so the time of the maximum of the second period's radial velocity should have been shifted by more than four months between the first and the last observing season. Inspite of this we re-analysed the measurements of Fekel et al. (2000). For the period analysis of the radial velocities we used the method of Fourier harmonic analysis (Andronov 1994), which fits the first harmonic term of a trigonometric polynomial to the data using the least squares method. For verification of the results the method of Fourier analysis (Deeming 1975; Kurtz 1985) was used.

The results of period analysis of AG Dra radial velocities taken from the original data of Fekel et al. (2000) are depicted in Fig. 1. In the corresponding periodogram, part (a) shows a few peaks, but the largest two of them are connected with the orbital period and with its 1-year alias. The orbital variations of radial velocity are described largely by two parameters: $P_{\text {orb }}=550.5$ days, $T_{\max }=$ JD 2451186.1 days. This orbital period is close to the value of $552.3 \pm 7.4$ days found by Fekel et al. (2000) from their observations. In addition we also found the second period in Fekel's data. The peak connected with this period is hard to see because of

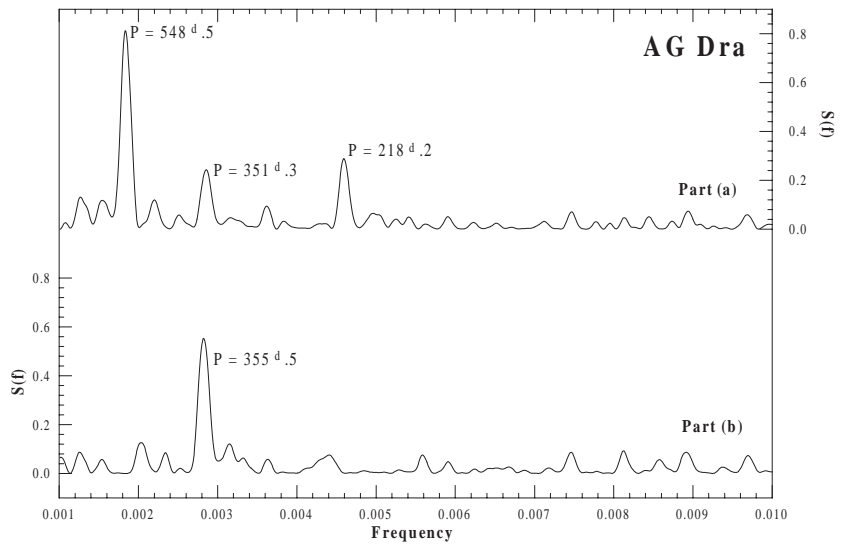

Fig. 2. Periodograms of AG Dra taken from combined data: original data - Part a) and orbital response removed data - Part b).

complex structure in the periodogram, there being two reasons: the insufficient amount of data and their inconvenient distribution. Nevertheless the second period is still present in this diagram and in our opinion it represents the pulsation of the cool component of AG Dra. The bottom periodogram (Fig. 1, part b) shows the result after removing the orbital response from the data. Inspite of larger noise the pulsation peak is clearly visible. The ephemeris of the pulsation variations of radial velocities are described by the following parameters: $P_{\text {pul }}=357.4$ days, $T_{\max }=$ JD 2451328.4 days. This pulsation period is in good agreement with our previous value (Gális et al. 1999). Moreover the value of $T_{\max }$ of the pulsation variations is only 2.2 days from that expected from the solution of Gális et al. (1999); this would suggest that despite the problem of the inconvenient distribution of the observations of Fekel et al. (2000), the second period found from the analysis of their independent measurements is real. All resulting parameters with their errors are listed in Table 1. The results based only on Fekel's data are presented in the second column for orbital response and in the third one for pulsation response respectively. It is interesting to discuss the larger value of semiamplitude in both cases. The orbital one is caused by two of Fekel's points near orbital phases 0.0 and 0.5 respectively. The pulsation value of this parameter is overestimated because of the problems mentioned above. In the last two lines of the table the sums of residual squares $(S R S)$ are derived as well as the corresponding standard errors of the data in order to show that the already low values of these parameters only solving for the orbital response are significantly lower after the pulsation response is taken into account.

In view of the last results we have combined the measurements of Fekel et al. (2000) with those used by Gális et al. (1999). The zero-point problem of separate radial velocity sets was solved by numerical minimization of the SRS of the observational points with respect to the synthetic curve of radial velocities (combined orbital and pulsation response) from our previous analysis. Fekel's data were shifted by the value $(1.5 \pm 0.6) \mathrm{km} \mathrm{s}^{-1}$ with respect to the data set used by Gális et al. (1999). The values of mean errors for particular sets were subtracted (independently from the results of the separate analysis of Fekel's data) from the analysis of both combined data sets as 
Table 1. Resulting parameters.

\begin{tabular}{|c|c|c|c|c|c|c|c|c|c|}
\hline \multirow{2}{*}{$\begin{array}{l}\text { Data file } \\
N\end{array}$} & \multicolumn{2}{|c|}{ 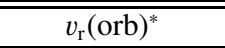 } & \multicolumn{2}{|c|}{ 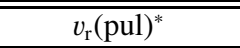 } & \multicolumn{2}{|c|}{$\overline{v_{\mathrm{r}}(\mathrm{orb})}$} & \multirow{2}{*}{$\begin{array}{l}v_{\mathrm{r}}(\text { orb }) \\
83\end{array}$} & \multicolumn{2}{|c|}{ 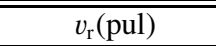 } \\
\hline & 10 & & 10 & & 83 & & & 83 & \\
\hline$P[\mathrm{~d}]$ & 550.5 & \pm 7.2 & 357.4 & \pm 3.8 & 548.5 & \pm 1.1 & 548.5 & 355.0 & \pm 1.5 \\
\hline$T_{\min }[\mathrm{JD} 24 .]$. & 51186.1 & \pm 4.4 & 51328.4 & \pm 11.4 & 48444.1 & \pm 3.1 & - & 48486.7 & \pm 6.9 \\
\hline$T_{0}[\mathrm{JD} 24 .]$. & - & & - & & - & & 48748.4 & - & \\
\hline$e$ & 0.0 & & - & & 0.0 & & 0.06 & - & \\
\hline$V_{0}\left[\mathrm{~km} \mathrm{~s}^{-1}\right]$ & -147.3 & \pm 0.2 & 3.5 & \pm 1.7 & -147.6 & \pm 0.1 & -147.6 & -147.5 & \pm 0.1 \\
\hline$\omega$ & - & & - & & - & & $359^{\circ} .8$ & - & \\
\hline$K_{1}\left[\mathrm{~km} \mathrm{~s}^{-1}\right]$ & 6.0 & \pm 0.3 & 4.1 & \pm 1.8 & 4.8 & \pm 0.2 & 4.7 & 1.7 & \pm 0.2 \\
\hline$A_{1} \sin i\left[R_{\odot}\right]$ & & & & & & & 51.1 & - & \\
\hline$f(M)\left[M_{\odot}\right]$ & & & & & & & 0.00595 & - & \\
\hline$S R S\left[\left(\mathrm{~km} \mathrm{~s}^{-1}\right)^{2}\right]$ & 2.4 & & 1.1 & & 96.3 & & 94.2 & 95.9 & \\
\hline$\sigma\left[\mathrm{km} \mathrm{s}^{-1}\right]$ & 0.516 & & 0.354 & & 1.084 & & 1.072 & 1.081 & \\
\hline
\end{tabular}

* The results of the analysis of the data taken only by Fekel et al. (2000).

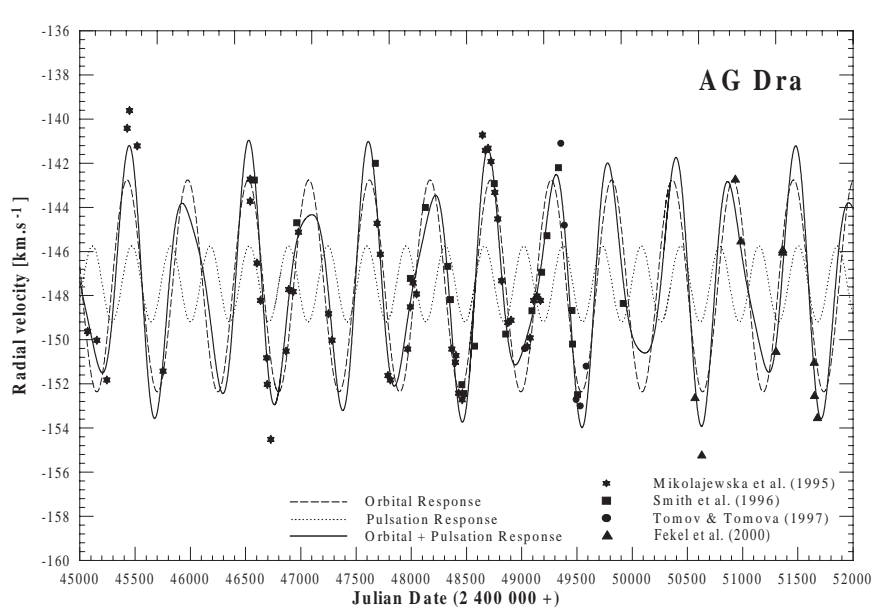

Fig. 3. Radial velocities and synthetic curves for particular responses presented.

follows: $1.1 \mathrm{~km} \mathrm{~s}^{-1}$ for the data set used by Gális et al. (1999) and $0.9 \mathrm{~km} \mathrm{~s}^{-1}$ for Fekel's data. With respect to similar values of these errors uniform weights were assigned to particular data sets. For the next analysis we used our own method (for more details see Gális et al. 1999) which consists of several iteration steps for particular responses. It is also worth noting that our iteration method has some advantages. The iterations of particular parameters can be used on small data sets too. The method requires only a minimal number of initial assumptions and therefore is able to discover manifestations of unknown phenomena in observational data. Moreover this method uses the precise values of search parameters as well as of their errors. One piece of evidence for these ideas is the good agreement of the values of orbital and pulsation parameters of AG Dra and their errors recently derived by an independent method for the same data sets by Van Hamme \& Wilson (2002).

After a few iteration steps improved parameters of orbital motion and pulsation have been obtained. The corresponding periodograms are depicted in Fig. 2. The final values of parameters are listed in Table 1: for orbital response in Col. 4 and for pulsation one in Col. 6. These parameters were used for constructing the synthetic radial velocity curves which are depicted in Fig. 3.

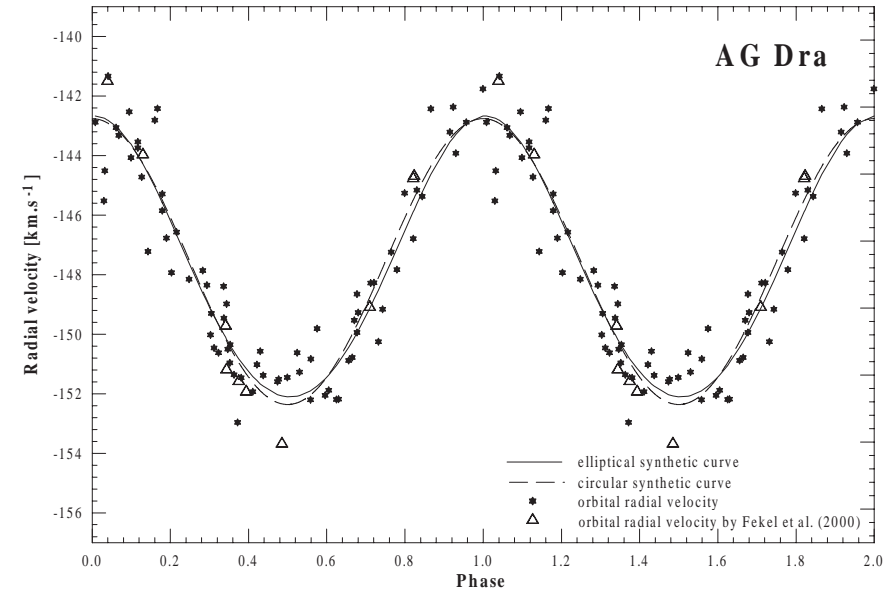

Fig. 4. Phase diagram of orbital radial velocities and comparison of circular and elliptical synthetic curves. Data by Fekel et al. (2000) are depicted individualy.

The final solution for the orbital elements was obtained by a program based on the simplex method (Kratka 1990), which is based on the optimization method of computation of the spectroscopic orbital elements. As input parameters we used the final values of orbital response from the iteration process with the pulsation response removed. The resulting orbital parameters are listed in Col. 5 of Table 1. It is shown in Fig. 4 that the fit of the orbital radial velocities to an elliptical orbit's synthetic curve is better in comparison with a circular synthetic one. In Fig. 5 the pulsation radial velocities are shown. The data derived from the radial velocities, published by Fekel et al. (2000), are depicted by triangles. Fekel's data are situated close to phase 0.5 , so unfortunately they do not cover the whole pulsation phase interval.

\section{Summary}

In this paper we re-analysed all available radial velocities of AG Dra. New more precise values of the orbital parameters of this binary system as well as the pulsation parameters of the cool red giant component are presented. Our high precision parameters, derived in this paper, confirmed the results published 


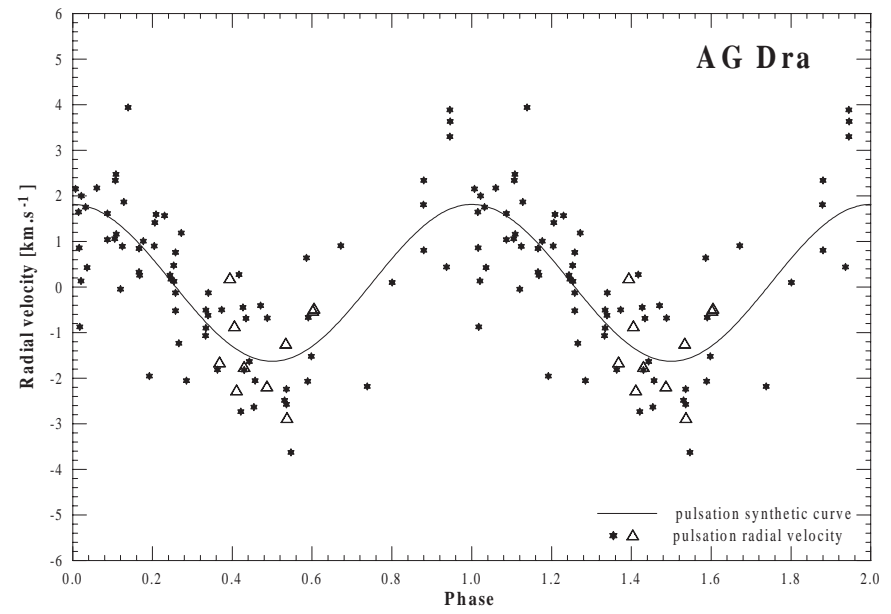

Fig. 5. Phase diagram of pulsation radial velocities and synthetic pulsation curve. Pulsation radial velocities derived from data by Fekel et al. (2000) are depicted by triangles.

in our previous paper (Gális et al. 1999). The results derived on the basis of all available data have been confirmed in Fekel's data set too and the second period caused by pulsation of cool component is also detectable in these data. Moreover we have found the reason for Fekel's inability to detect pulsation response. Basically, the phase distribution of Fekel's 10 radial velocities contracts to very narrow phase interval of pulsation (close to 0.5) and their analysis failed. On the other hand fully distributed data including Fekel's ones clearly showed both, i.e. orbital and pulsation variations, respectively.

We can conclude that the presence of pulsations of the cooler component in radial velocity data is still a plausible explanation. These conclusions are moreover strengthened and theoretical work is required to better understand the exact nature of the second period. In any case more radial velocity measurements will be required at later dates to lift any remaining doubts.

Acknowledgements. This work has been partly supported through the Slovak Academy of Sciences Grant No. 1008/21, through STAA Grant No. 51-000802 as well as by Faculty of Education, University of Trnava Grant No. 02/02.

\section{References}

Andronov, I. L. 1994, Odessa Astronomical Publications 7, part 1, 49 Bastian, U. 1998, A\&A, 329, L61

Deeming, T. J. 1975, Ap\&SS, 36, 137

Friedjung, M., Hric, L., Petrík, K., \& Gális, R. 1998, A\&A, 335, 545

Fekel, F. C., Hinckle, K. H., Joyce, R. R., \& Skrutskie, M. F. 2000, AJ, 120, 3255

Gális, R., Hric, L., Friedjung, M., \& Petrík, K. 1999, A\&A, 348, 533

Kenyon, S. J., \& Fernandez-Castro, T. 1987, AJ, 93, 938

Kratka, M. 1990, private comm.

Kurtz, O. W. 1985, MNRAS, 213, 773

Mikolajewska, J., Kenyon, S. J., Mikolajewski, M., Garcia, M. R., \& Polidan, R. S. 1995, AJ, 109, 1289

Petrík, K., Hric, L., Gális, R., Friedjung, M., \& Dobrotka, A. 1998, IBVS, 4588

Smith, V. V., Cunha, K., Jorissen, A., \& Boffin, H. M. J. 1996, A\&A, 315,179

Tomov, N. A., \& Tomova, M. T. 1997, in Physical Processes in Symbiotic Binaries and Related Systems, ed. J. Mikolajewska, Copernicus Foundation for Polish Astronomy, Warsaw, 185

Van Hamme, W., \& Wilson, R. E. 2002, in Exotic Stars as Challenges to Evolution, ed. C. A. Tout, \& W. Van Hamme (San Francisco: ASP), ASP Conf. Ser., 279, 161

Zhu, Z. X., Friedjung, M., Zhao, G., Hang, H. R., \& Huang, C. C. 1999, A\&A, 140, 69 